\title{
Second-hand smoke exposure in cars and respiratory health effects in children
}

\author{
Z. Kabir*, P.J. Manning\#, J. Holohan`, S. Keogan*, P.G. Goodman ${ }^{+}$and L. Clancy*
}

ABSTRACT: We examined potential associations of ever asthma, and symptoms of wheeze (past 12 months), hay fever, eczema and bronchitis (cough with phlegm) among school children exposed to second-hand smoke (SHS) in cars, using a modified Irish International Study of Asthma and Allergies in Childhood (ISAAC) protocol.

2,809 children of 13-14 yrs old and who selected randomly from post-primary schools throughout Ireland completed the 2007 ISAAC self-administered questionnaire. Adjusted OR (adjusted for sex, active smoking status of children interviewed and their SHS exposure at home) were estimated for the associations studied, using multivariable logistic regression techniques.

Overall, $14.8 \%$ (13.9\% in young males, $15.4 \%$ in young females) of Irish children aged $13-14$ yrs old were exposed to SHS in cars. Although there was a tendency towards increased likelihood of both respiratory and allergic symptoms with SHS exposure in cars, wheeze and hay fever symptoms were significantly higher (adjusted OR 1.35 (95\% Cl 1.08-1.70) and 1.30 (1.01-1.67), respectively), while bronchitis symptoms and asthma were not significant (1.33 (0.92-1.95) and 1.07 (0.81-1.42), respectively).

Approximately one in seven Irish schoolchildren are exposed to SHS in cars and could have adverse respiratory health effects. Further studies are imperative to explore such associations across different population settings.

KEYWORDS: Asthma, bronchitis, cars, children, Ireland, smoking

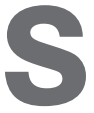
econd-hand smoke (SHS) is a group 1 carcinogen; there is no safe level of SHS exposure [1]. Considering that children are relatively more exposed to SHS than adults [2] and are less likely to prevent their own exposure, children are at greater risk of future SHS-related morbidity and mortality. The adverse health effects of SHS exposure among nonsmoking children include upper and lower respiratory tract infections (e.g. bronchitis and pneumonia), asthma, otitis media, and sudden infant death syndrome [3-5]. Children exposed to SHS also report more days of restricted activity, more days of bed confinement, and more days of school absence than those not exposed to SHS [6]. Exposure of children to SHS in cars increases the risk of nicotine dependence symptoms [7]. Reducing childhood SHS exposure should be a public health priority.

Children may be more vulnerable to SHS-induced respiratory diseases due to smaller airways and greater oxygen demand, as well as less mature immune systems [8]. There is no evidence quantifying SHS-induced respiratory health effects in children exposed to SHS in cars. Three recent studies that examined childhood SHS exposure in cars reported levels of $6.5 \%$ in the UK, $26.3 \%$ in Canada, and $40.2 \%$ in Nebraska [9-11]. Ireland introduced a comprehensive workplace smoking ban in March 2004 [12], with positive health effects being reported post ban $[12,13]$. At present, the two domains that offer the best intervention potential are homes and cars. SHS in a car is 23 times more toxic than in a house due to the enclosed space [14]. With this information in mind, the present study had the following objectives: 1) to estimate the prevalence of childhood SHS exposure in cars among Irish schoolchildren aged 13-14 yrs old; and 2) to examine an association between childhood SHS exposure in cars and five respiratory health effects (asthma, wheeze, bronchitis symptoms, hay fever, and eczema) among Irish schoolchildren aged 13-14 yrs old.

\section{METHODS}

In 2007, we undertook a local-level International Study of Asthma and Allergies in Childhood
AFFILIATIONS

${ }^{\star}$ Tobacco Free Research Institute,

${ }^{*}$ Respiratory Medicine, St. James's Hospital,

"Asthma Society of Ireland, and

${ }^{+}$Dublin Institute of Technology,

Dublin, Ireland

CORRESPONDENCE

L. Clancy

Tobacco Free Research Institute

The Digital Depot

Thomas Street

Dublin 8

Ireland

E-mail: Iclancy@tri.ie

Received:

Nov 052008

Accepted after revision:

March 082009

First published online:

April 082009 
(ISAAC) follow-up study using the ISAAC questionnaire [15, 16] and as part of that study we included an additional question on SHS exposure in cars. The ISAAC sample is a nationally representative sample. Details of the sampling design of the ISAAC studies are described elsewhere [15, 16].

In brief, the population of interest was schoolchildren in Ireland, aged 13-14 yrs old. Schoolchildren in this age range were chosen to comply with the ISAAC study requirements. From each school, classes with the greatest proportion of 13$14 \mathrm{yr}$ olds were selected. The basic sampling frame consisted of all post-primary schools $(n=731)$, excluding specialised disability schools and those with $<40$ students because of the impracticalities of administering the survey. Schools were selected by stratified random sampling based on school size and composition. They were also stratified by Health Board Areas (now known as the single Health Services Executive) to allow for regional comparisons of the data. A pre-study sample size of $\geqslant 3,000$ children was calculated in a similar manner to the design of a previous study which examined respiratory symptoms in Ireland [17]. The data collection was carried out between March and April 2007 before commencement of the main grass and tree pollen season. The questionnaires were self-administered under the supervision of the researchers. A participation rate of $90 \%$ was aimed for, and efforts were made to follow up students who were absent on the day the questionnaires were administered. School registers were checked for student absentees on the day of the survey and provision was made for completed questionnaires from these students to be returned by post. Of the 39 schools invited, 35 schools participated in this survey. The total sample size was 3,052 respondents, with 2,809 of these being included in the final analyses; the others were excluded due to being outside the required age group of 13-14 yrs.

\section{Health outcomes}

In a similar manner to previous studies [15-18], asthma was defined as ever asthma and was self-reported. Bronchitis symptoms included having both cough with phlegm during the previous 12 months. Asthmatics were excluded when considering bronchitic symptoms, reducing the sample size to 2,278 for this particular health outcome. Hay fever and eczema were self-reported, as ever hay fever and ever eczema. Wheeze symptoms were ascertained using the question: "Have you had wheezing or whistling in the chest in the last 12 months? Yes/ $\mathrm{No}^{\prime \prime}$. Details on the ISAAC questionnaire are presented in the online supplementary material.

\section{SHS exposure in cars and at home}

SHS exposure in cars was based on the self-reported question: "If you travel to school by car, does anyone smoke cigarettes in the car? Yes/No". SHS exposure at home was assessed using the question: "Does anyone you live with smoke cigarettes regularly at home? Yes/No". There were also boxes referring to those who smoke: mother/father/siblings/others. No objective validation of SHS exposure was performed; however, evidence suggests that self-reported SHS exposure correlates strongly with objective measurements, such as serum cotinine level measurements [19].

\section{Statistical analyses}

Both descriptive and multivariable logistic regression analyses were performed using SAS software (version 9.1; SAS Institute Inc., Cary, NC, USA). In order to identify whether there was an interaction between SHS exposure at home and SHS exposure in cars across the five respiratory health effects, stratumspecific estimates were computed, stratifying child exposure to SHS at home into two sub-groups (exposed and not exposed). The stratum-specific estimates were different; therefore, an interaction term was applied to each of the multivariable logistic regression models for tests of interaction. However, in the final models, the interaction terms were removed as they were not statistically significant.

An unadjusted estimate for each of the health outcomes was computed, followed by adjustment for active smoking status of the children alone. Next, adjustment for SHS exposure at home was performed. Finally, for all of the potential confounders available in the study (sex, current smoking status of the children, SHS exposure at home), we simultaneously adjusted for the associations examined to compute adjusted ORs (AOR) using multivariable logistic regression techniques. The Hosmer-Lemeshow goodness-of-fit model was applied [20]. All models had a statistical significance of $\mathrm{p}>0.05$.

\section{RESULTS}

Overall, $14.8 \%$ (417 out of 2,809) of Irish children aged 1314 yrs old were exposed to smoking in cars. More young females $(15.4 \%)$ were exposed to SHS in cars than young males (13.9\%). Table 1 shows the prevalence of various respiratory health effects (asthma, wheeze symptoms, bronchitis symptoms, hay fever and eczema), comparing those exposed to SHS in cars to those not exposed to SHS in cars. Almost $52 \%$ of young females exposed to SHS in cars had wheeze symptoms, compared to $38 \%$ of young females who suffered from wheeze symptoms if not exposed to SHS in cars $(p<0.0001)$. No statistically significant increases in ever asthma prevalence rates were observed overall $(p=0.57)$ nor were there any statistically significant sex differences in ever asthma rates when children were exposed to SHS in cars. $16 \%$ of young females, with no history of asthma, suffered bronchitis symptoms when exposed to SHS in cars; comparatively, $8 \%$ of young females had bronchitis symptoms if not exposed to SHS in cars $(p=0.0002)$. More than $21 \%$ of young females also suffered from eczema if exposed to SHS in cars, compared to $15.3 \%$ not exposed $(p=0.01)$. Overall, hay fever symptoms were significantly more common among children exposed to SHS in cars $(\mathrm{p}=0.03)$.

Table 2 shows the multivariable logistic regression analyses for the associations between childhood SHS exposure in cars and the five health effects studied. When simultaneously adjusting for sex, current smoking status and SHS exposure at home, children exposed to SHS in cars showed a 35\% increased risk of having wheeze symptoms (AOR 1.35, 95\% CI 1.08-1.70). Such increased wheeze symptoms persisted when individuals with asthma were also accounted for (AOR 1.40, 95\% CI 1.08-1.80). An increased risk of hay fever symptoms was observed among children exposed to SHS in cars (AOR 1.30, 95\% CI 1.01-1.67). When non-asthmatics with bronchitis symptoms were analysed, the estimates did not reach statistical significance (AOR $1.33,95 \%$ CI $0.92-1.95$ ) despite showing a two-fold increased 


\begin{tabular}{|c|c|c|c|c|c|c|c|c|c|c|c|c|c|c|c|}
\hline \multirow[t]{3}{*}{ TABLE } & \multicolumn{15}{|c|}{$\begin{array}{l}\text { Prevalence of respiratory health effects in children aged 13-14 yrs old exposed and not exposed to second-hand } \\
\text { smoke (SHS) in cars in Ireland }\end{array}$} \\
\hline & \multicolumn{3}{|c|}{ Asthma ${ }^{\#}$} & \multicolumn{3}{|c|}{ Wheeze $^{\#}$} & \multicolumn{3}{|c|}{ Bronchitis } & \multicolumn{3}{|c|}{ Hay fever ${ }^{\#}$} & \multicolumn{3}{|c|}{ Eczema $^{\#}$} \\
\hline & SHS & No SHS & $p$-value & SHS & No SHS & $p$-value & SHS & No SHS & $p$-value & SHS & No SHS & $\mathrm{p}$-value & SHS & No SHS & p-value \\
\hline Overall & 19.9 & 18.7 & 0.57 & 48.2 & 37.4 & 0.0001 & 14.7 & 7.7 & 0.0001 & 29.5 & 24.5 & 0.03 & 15.8 & 12.7 & 0.08 \\
\hline Males & 20.9 & 19.9 & 0.78 & 41.8 & 35.6 & 0.14 & 12.0 & 6.9 & 0.047 & 28.5 & 23.1 & 0.14 & 6.9 & 9.0 & 0.39 \\
\hline Females & 19.4 & 17.9 & 0.58 & 51.9 & 38.6 & 0.0001 & 16.3 & 8.2 & 0.0002 & 30.2 & 25.4 & 0.10 & 21.3 & 15.3 & 0.01 \\
\hline
\end{tabular}

risk when not adjusted for both SHS at home and active smoking status (AOR 2.06, 95\% CI 1.46-2.92). Childhood asthma and eczema are least likely to be associated with SHS exposure in cars (table 2).

\section{DISCUSSION}

To our knowledge, this is the first epidemiological study to examine potential associations of respiratory health effects in children exposed to involuntary SHS in cars across a nationally representative sample of 13-14 yr olds. Although the observations are unlikely to be due to chance, the study findings are to be interpreted with caution. No similar evidence is available elsewhere with which to compare the results. Secondly, misclassification bias in exposure assessment is always a possibility in a cross-sectional survey. Thirdly, residual confounding might be a possibility due to some unmeasured/unidentified confounders.

At $14.8 \%$, the self-reported childhood SHS exposure in cars in Ireland is higher than that reported in pupils (mean age $11.4 \mathrm{yrs}$ ) in Scotland 1-yr after the Scottish smoking ban in 2006 $(6.5 \%)$ [9], but lower than the reported prevalence among 12 and 17 yr olds in Canada where a similar ban was not in place $(26.3 \%)$ [10]. SHS exposure in cars might also indirectly reflect the "heavy" smoking habits of those smoking at home. Unfortunately, the Irish ISAAC 2007 question on SHS exposure in cars does not make such distinctions and thus could not be accounted for in the analysis phase (see online supplementary material for the ISAAC questionnaire, reproduced with permission from ISAAC (http://isaac.aukland.ac.nz). In the present study, young females were significantly more exposed to SHS in cars. Young females exposed to SHS in cars also had significantly increased odds of suffering from both wheeze and bronchitis symptoms when compared to males of a similar age. However, the present study showed that childhood asthma is not statistically associated with SHS exposure in cars.

Similar to all cross-sectional surveys, the present study has methodological limitations additional to those previously mentioned. Smoking history (SHS both in cars and at home, and active smoking) and health effects are self-reported, and this might introduce recall and misclassification biases. Such exposure misclassification bias would be non-differential, further pushing the ORs towards null estimates. Empirical evidence also suggests that objective measurements using serum cotinine levels correlate well with self-reported smoking history [19]. Furthermore, a causal inference cannot be drawn based on the cross-sectional design of this study. Taken together, comprehensive longitudinal long-term follow-up studies are imperative. However, the strength of the present study is the limited chance of a selection bias because of random probability sampling technique. Therefore, any significant findings observed could be generalised to all of the Irish schoolchildren and to comparable populations elsewhere in Europe.

TABLE 2 Logistic regression models for the associations between childhood second-hand smoke (SHS) exposure in cars and respiratory health effects among Irish schoolchildren aged 13-14 yrs old

AOR $(95 \% \mathrm{Cl})$

Unadjusted OR $(95 \% \mathrm{Cl})^{\S}$

\begin{tabular}{|c|c|c|c|c|}
\hline & Both $^{\#}$ & Passive only & Active only ${ }^{+}$ & \\
\hline Asthma & $1.07(0.81-1.42)$ & $1.06(0.80-1.41)$ & $1.09(0.84-1.42)$ & $1.08(0.83-1.41)$ \\
\hline Bronchitis & $1.33(0.92-1.95)$ & $1.42(0.98-2.07)$ & $1.75(1.23-2.51)$ & $2.06(1.46-2.92)$ \\
\hline Hay fever & $1.30(1.01-1.67)$ & $1.32(1.02-1.69)$ & $1.27(1.01-1.60)$ & $1.29(1.03-1.63)$ \\
\hline Eczema & $1.24(0.90-1.70)$ & $1.27(0.92-1.74)$ & $1.22(0.91-1.64)$ & $1.27(0.95-1.70)$ \\
\hline
\end{tabular}

Values shown in bold are statistically significant. AOR: adjusted OR. ${ }^{\#}$ : adjusted for sex, current smoking and SHS exposure at home; ${ }^{\circ}$ : adjusted for sex and SHS exposure at home; ${ }^{+}$: adjusted for sex and current smoking; ${ }^{\text {s: }}$ adjusted for sex only. 
Aside from negative health effects, evidence suggests that children exposed to SHS have an increased likelihood of starting smoking [21]. Some $80 \%$ of adult smokers started smoking before 18 yrs of age [22]. Therefore, reducing childhood SHS exposure should be a public health priority. Homes and cars are the principal sources of children's SHS exposure. A recent Irish study reported $41 \%$ SHS exposure levels at home nationwide [23]. An Action on Smoking Health (ASH) Ireland survey performed recently reported that almost $79 \%$ of respondents supported legislation against smoking in private vehicles if children are present [24]. Before contemplating moving forward with programmes and/or policies designed to reduce or eliminate SHS exposure among children in cars based on these health findings alone, further explorations are needed.

In several places, moves have been initiated to institute smokefree cars (for example, when children are present) based on a few studies in Canada [25] and in the USA [26] that reported very high levels of pollution due to SHS in cars. Despite such improvements in a few countries worldwide, the fact that a substantial number of children continue to be exposed to SHS indicates that more needs to be done in terms of SHS prevention programming. However, little is known about which programmes are effective [27].

\section{Conclusion}

One in seven Irish schoolchildren is exposed to SHS in cars. Despite the fact that the present study shows a tendency towards an increased likelihood of respiratory and allergic symptoms in children when exposed to SHS in cars, comprehensive longitudinal studies across different population settings are imperative. Assuming a causal relation, such adverse respiratory symptoms could have a knock-on effect on school absenteeism, and on a greater risk for future SHSrelated morbidity and mortality. Evidence suggests that children exposed to SHS in places other than homes are more likely to be susceptible to starting smoking than those not exposed [28], and children particularly exposed to SHS in motor vehicles have nicotine-dependence symptoms [7]. These results further support efforts to push ahead with legislation supporting smoke-free cars in Ireland; however, they need to be adequately substantiated with further evidence from elsewhere.

\section{SUPPORT STATEMENT}

Z. Kabir, L. Clancy and S. Keogan are employees of the Tobacco Free Research Institute, a non-profit organisation funded in part through a grant from the Department of Health and Children (Ireland). J. Holohan is the Chief Executive of the Asthma Society, a non-profit organisation. The present study's findings are independent of organisational influences. The Royal City of Dublin Hospital Trust (Dublin, Ireland) provided financial support for the present study.

\section{STATEMENT OF INTEREST}

None declared.

\section{ACKNOWLEDGEMENTS}

The authors would like to acknowledge the following groups: the Board of Asthma Society of Ireland; the pupils, parents, teachers and principals of the schools involved in the study; and the Respiratory Nurses Association of Ireland (ANAIL; Dublin, Ireland) who carried out the research.

\section{REFERENCES}

1 International Agency for Research on Cancer. IARC Monographs on the Evaluation of Carcinogenic Risks to Humans: Tobacco Smoke and Involuntary Smoking. Vol. 83. Lyon, IARC, 2004.

2 Centers for Disease Control and Prevention. Third national report on human exposure to environmental chemicals. Atlanta, US Department of Health and Human Services, 2005.

3 Gürkan F, Kiral A, Dağli E, et al. The effect of passive smoking on the development of respiratory syncytial virus bronchiolitis. Eur J Epidemiol 2000; 16: 465-468.

4 Adair-Bischoff CE, Sauve RS. Environmental tobacco smoke and middle ear disease in preschool-age children. Arch Pediatr Adolesc Med 1998; 152: 127-133.

5 Mitchell EA, Ford RP, Stewart AW, et al. Smoking and the sudden infant death syndrome. Pediatrics 1993; 91: 893-896.

6 Mannino DM, Siegel M, Husten C, et al. Environmental tobacco smoke exposure and health effects in children: results from the 1991 National Health Interview Survey. Tob Control 1996; 5: 13-18.

7 Bélanger M, O'Loughlin J, Okoli CT, et al. Nicotine dependence symptoms among young never-smokers exposed to secondhand tobacco smoke. Addict Behav 2008; 33: 1557-1563.

8 Elliot J, Vullermin P, Robinson P. Maternal cigarette smoking is associated with increased inner airway wall thickness in children who die from sudden infant death syndrome. Am J Respir Crit Care Med 1998; 158: 802-806.

9 Leatherdale ST, Smith P, Ahmed R. Youth exposure to smoking in the home and in cars: how often does it happen and what do youth think about it? Tob Control 2008; 17: 86-92.

10 Akhtar PC, Currie DB, Currie CE, et al. Changes in child exposure to environmental tobacco smoke (CHETS) study after implementation of smoke-free legislation in Scotland: national cross sectional survey. BMJ 2007; 335: 545.

11 Mbulo L. Changes in exposure to secondhand smoke among youth in Nebraska, 2002-2006. Prev Chronic Dis 2008; 5: A84.

12 Goodman P, Agnew M, McCaffrey M, et al. Effects of the Irish smoking ban on respiratory health of bar workers and air quality in Dublin pubs. Am J Respir Crit Care Med 2007; 175: 840-845.

13 Allwright S, Paul G, Greiner B, et al. Legislation for smoke-free workplaces and health of bar workers in Ireland: before and after study. BMJ 2005; 331: 1117.

14 Ontario Medical Association. Exposure to secondhand smoke: are we protecting our kids? Toronto, Ontario Medical Association, 2005.

15 Manning PJ, Curran K, Kirby B, et al. Asthma, hay fever and eczema in Irish teenagers (ISAAC protocol). Ir Med J 1997; 90: 110-112.

16 Manning PJ, Goodman P, O'Sullivan A, et al. Rising prevalence of asthma but declining wheeze in teenagers (1995-2003): ISAAC protocol. Ir Med J 2007; 100: 614-615.

17 Yarnell JW, Stevenson MR, MacMahon J, et al. Smoking, atopy and certain furry pets are major determinants of respiratory symptoms in children: the International Study of Asthma and Allergies in Childhood Study (Ireland). Clin Exp Allergy 2003; 3: 96-100.

18 Al-Rawas OA, Al-Riyami BM, Al-Maniri AA, et al. Trends in asthma prevalence and severity in Omani schoolchildren: comparison between ISAAC phases I and III. Respirology 2008; 13: 670-673.

19 Gilpin EA, Pierce JP, Cavin SW, et al. Estimates of population smoking prevalence: self vs proxy reports of smoking status. Am J Public Health 1994; 84: 1576-1579.

20 Hosmer DW, Lemeshow S. Applied logistic regression. New York, Wiley, 2000.

21 Bettcher DW, Peruga A, Fishburn B, et al. Exposure to secondhand smoke among students aged 13-15 years-worldwide, 2000-2007. MMWR 2007; 56: 497-500.

22 U.S. Department of Health and Human Services. Preventing tobacco use among young people: A report of the Surgeon General. Atlanta, U.S. Department of Health and Human Services, 1994. 
23 ASH Ireland TNBRI survey. www.ash.ie/News/Latest_News/ ASH_call_for_Smoking_Ban.html Date last accessed: April 4, 2007. Date last updated: June 2009.

24 Morgan K, McGee H, Watson D, et al. SLAN 2007: Survey of Lifestyle, Attitudes and Nutrition in Ireland. Main Report. Dublin, Department of Health and Children, 2008.

25 Health Canada. Make Your Home and Car Smoke-Free: a Guide to Protecting Your Family from Second-Hand-Smoke. Ottawa, Health Canada, 2006.
26 Rees VW, Connolly GN. Measuring air quality to protect children from secondhand smoke in cars. Am J Prev Med 2006; 31: 363-368.

27 Hovell MF, Zakarian JM, Wahlgren DR, et al. Reducing children's exposure to environmental tobacco smoke: the empirical evidence and directions for future research. Tob Control 2000; 2: II40-II47.

28 Centers for Disease Control and Prevention. Exposure to secondhand smoke among students aged 13-15 years - worldwide, 20002007. Morb Mortal Wkly Rep 2007; 56: 497-500. 